\title{
BMJ Open Understanding on the association between informal caregiver characteristics and cognitive function of adults with cancer: a scoping review protocol
}

\author{
Yesol Yang, ${ }^{0}$ Sharron Rushton, ${ }^{2}$ Amanda Woodward, ${ }^{3}$ Cristina Hendrix ${ }^{2,4}$
}

To cite: Yang Y, Rushton S,

Woodward A, et al. Understanding on the association between informal caregiver characteristics and cognitive function of adults with cancer: a scoping review protocol. BMJ Open 2019;9:e031842. doi:10.1136/ bmjopen-2019-031842

- Prepublication history and additional material for this paper are available online. To view these files, please visit the journal online (http://dx.doi. org/10.1136/bmjopen-2019031842).

Received 22 May 2019 Revised 04 September 2019 Accepted 04 September 2019

\section{Check for updates}

(C) Author(s) (or their employer(s)) 2019. Re-use permitted under CC BY-NC. No commercial re-use. See rights and permissions. Published by BMJ.

${ }^{1}$ Duke University School of Nursing, Durham, North Carolina, USA

${ }^{2}$ Division of Health Systems and Analytics, Duke University School of Nursing, Durham, North Carolina, USA

${ }^{3}$ Duke University Medical Center Library, Durham, North Carolina, USA

${ }^{4}$ GRECC, Durham Veteran Affairs Health Care System, Durham, North Carolina, USA

Correspondence to Yesol Yang;

yesol.yang@duke.edu

\section{ABSTRACT}

Introduction Adults with cancer frequently report symptoms such as decline in cognitive function throughout the trajectory of illness. Patients with cognitive deficits need support and assistance from their informal caregivers and often rely on them to manage their symptoms based on their degree of deficits. Patients spend a significant amount of time with their informal caregivers and become interdependent with each other. In spite of their interdependence, it is unclear whether patients' cognitive outcomes (ie, cognitive function) are associated with their informal caregivers. Therefore, the body of literature related to the association between caregiver characteristics and cognitive function of adults with cancer needs to be fully mapped with assessment for knowledge gaps.

Methods and analysis Methods for this scoping review was informed by the framework proposed by Arksey and 0'Malley. Seven electronic databases will be searched: (1) PubMed (MEDLINE), (2) CINAHL, (3) Embase, (4) PsycINFO, (5) Scopus, (6) Sociological Abstracts and (7) ProQuest dissertation abstracts. In addition, the search for grey literature will include the conference abstracts available through Embase, Scopus and Sociological abstracts as well as dissertations available in ProQuest dissertations. All retrieved citations will be independently screened by two authors and eligibility will be determined based on inclusion and exclusion criteria at title and abstract level. Studies meeting inclusion criteria, will be screened at full text level by two reviewers followed by abstraction of included studies. Eligible studies will be collated, summarised and reported using the data charting form that research team developed.

Ethics and dissemination This scoping review does not require ethics approval. Results of this scoping review will be disseminated via conference presentation and/or publication in a scientific journal.

\section{BACKGROUND}

Cognitive deficits are frequently reported by adults with cancer. They can be defined as a decline in one or more domains of cognitive function ${ }^{1}$ : (1) attention, (2) concentration, (3) information processing speed, (4)
Strengths and limitations of this study

- This will be the first scoping review on the association between informal caregiver characteristics and cognitive function of adults with cancer.

- Using a scoping review methodology will allow review and mapping of literature from multiple disciplines and sources on informal caregiver characteristics within the context of cognitive function of adult cancer patients.

- Since the main purpose of conducting scoping review is to map the knowledge on a specific topic, a quality assessment of articles will not be performed. - Non-English studies will not be eligible for inclusion.

memory, (5) language, (6) executive function, (7) spatial ability and (8) psychomotor ability. Up to $75 \%$ of patients on cancer treatments have experienced cognitive deficits. Of those, $35 \%$ have reported that these deficits persist even after the completion of cancer treatments. ${ }^{2}$ The cognitive deficits can negatively influence the patient's social relationship with family or friends, overall life satisfaction and adherence to treatment. ${ }^{34}$

Cognitive deficits can develop regardless of cancer types throughout the trajectory of illness.

Although most studies on cognitive deficits have focused on women with breast cancer, ${ }^{5-7}$ these deficits are also found among adults with other types of cancer such as testicular cancer, ${ }^{8}$ lymphoma, ${ }^{9}$ ovarian cancer $^{10}$ and prostate cancer. ${ }^{11}$ Those patients appear to experience similar manifestations/trajectories of cognitive deficits as breast cancer patients do. However, despite its similarities between each group, no studies exist that have broadly explored cognitive deficits that occur across cancer types. 
Cognitive deficits in adults with cancer are most often multifactorial. A review of empirical literature revealed that (1) cancer process itself and/or (2) adverse effect from cancer treatments (eg, fatigue, mood or sleep disturbance) can contribute to patients' cognitive deficits. ${ }^{12}$ Also, some researchers have noted patients' age, ${ }^{13} 14$ genetic variations ${ }^{1516}$ or level of education ${ }^{17}$ as contributing factors to their cognitive deficits. However, these factors do not sufficiently explain the variabilities in the manifestations of patients' cognitive deficits. For example, some patients have reported relatively mild and shorter duration of cognitive deficits. ${ }^{518}$ Such inconsistencies in the manifestations of cognitive deficits heighten the need to investigate other potential factors, whether internal or external, that can explain the clinical variabilities.

Caregiver characteristics may provide some explanation on the clinical variabilities in cognitive function of adults with cancer. A study reported that when caring for patients with cognitive deficits, caregivers often expressed frustration in situations that required them to repeat same things due to their patients forgetfulness. ${ }^{3}$ Patients who witnessed their caregivers' frustration also reported frustration and embarrassment, ${ }^{3}$ and such patients' mood disturbances could contribute to their cognitive deficits. ${ }^{12}$ Von $\mathrm{Ah}$ and colleagues reported that patients experienced less confidence in themselves and mood disturbances when their family noticed their cognitive deficits. ${ }^{4}$ Furthermore, patients emphasised the importance of family/caregiver support on their cognitive function stating that support from their caregivers may buffer the negative consequence of their cognitive deficits. ${ }^{19}$

While the full scope of the association between patient's cognitive outcomes (ie, cognitive function) and their informal caregiver's characteristics still remains unclear, some literature identified that there may be an association between the two. Thus, clear understanding the association between informal caregiver characteristics and patient's cognitive function will be needed and this would help design more effective dyadic interventions. Several cancer studies suggest that a dyadic intervention can create synergistic effects that are more effective than treating the patient alone..$^{20-22}$ In summary, this understanding can inform the development of more precise and effective dyadic interventions that will improve outcomes for both patients and their informal caregivers in the context of cancer.

\section{Study rationale}

Several neurocognitive studies have reported informal caregiver characteristics are associated with patients' cognitive deficits. Specifically, caregiver burden, ${ }^{23-25}$ psychological well-being, ${ }^{26}$ coping strategies, ${ }^{27}{ }^{28}$ personalities $^{29}$ or caregiving relationship ${ }^{30}$ were found having an association with the level of patients' cognitive function. Of those, four characteristics such as caregiver burden, ${ }^{25}$ coping, ${ }^{28}$ personalities ${ }^{29}$ and caregiving relationship ${ }^{30}$ have been noted to predict patients' cognitive trajectories. However, despite the association between informal caregiver characteristics and patients' cognitive function, it remains unclear whether caregiver characteristics drive the association with cognitive function of adults with cancer. Further, no existing published review articles on a given association were identified from Prospero, PubMed (MEDLINE), CINAHL, Embase, PsycINFO, Scopus, Sociological Abstracts and ProQuest dissertation abstracts.

\section{Study objectives}

This scoping review will be undertaken to map the literature showing the association between informal caregiver characteristics and cognitive function of adults with cancer, determine the volumes of studies that are available and fill the knowledge gap. ${ }^{31}$ Furthermore, this scoping review may guide future research and/or identify a knowledge base for developing dyadic interventions that could be used in clinical setting. To accomplish these aims, this protocol manuscript is critical because it warrants the transparency and validity of the methods. The aim of protocol for a scoping review is to systematically review the relevant literature on informal caregiver characteristics associated with cognitive function of people with cancer.

\section{METHODS AND ANALYSIS Conceptual framework}

The scoping review will be guided by a model of reciprocal relationship between patient and caregiver emotional distress, ${ }^{32}$ which has been created based on two meta-analyses. ${ }^{33} 34$ In this model, when adults with cancer demonstrated high level of distress, their caregivers' level of distress also increased, and vice versa. This model provides the conceptual underpinning of viewing patient-caregiver dyad as the unit of care. Although this model has primarily focused on the association between patients' and caregivers' distress, we will adopt it to explore the association between patients' cognitive function and caregiver characteristics.

\section{Protocol design}

Methods for this scoping review is guided by the framework proposed by Arksey and O'Malley ${ }^{35}$ which has been further developed by Levac and colleagues. ${ }^{36}$ According to Arksey and O'Malley's framework, there are five methodological stages to follow:

Stage 1: identifying the research question.

Stage 2: identifying relevant studies.

Stage 3: selecting studies.

Stage 4: charting the data.

Stage 5: collating, summarising and reporting the results.

\section{Stage 1: Identifying the research question}

Based on Levac and colleagues' methodology for scoping reviews with using the PCO model,${ }^{36}$ we identified one overarching research question (P: population; C: concept; 
O: outcome), with 'P' representing the study population (adults with cancer), 'C', concept (caregiver characteristics) and 'O', outcome (cognitive function of adults with cancer). Using the PCO technique, we have elaborated one research question: 'What caregiver characteristics are associated with cognitive function of adults with cancer?' We limited our study population of patients to adult groups ( $\geq 18$ years old) because of different trajectories and manifestations of cognitive deficits that children show compared with adults with cancer. ${ }^{37-39}$ Also, we included informal caregivers who were at least 18 years old because caregivers aged less than 18 years may be more vulnerable to caregiving burden. ${ }^{40}$ In order to capture the full breadth of existing literature, we did not limit our study population to a specific cancer type.

\section{Stage 2: Identifying relevant studies}

We will employ an iterative process for identifying systematic search strategy to comprehensively address the research questions. Our review team will work closely with a librarian to develop relevant search key terms and its strategy. The librarian on our team will play a key role in determining and testing appropriate key terms, Medical Subject Headings (MeSH) terms and filters to maximise sensitivity and specify within the search. To ensure that all relevant information is captured, the authors will screen titles, abstracts, and index terms of the retrieved articles from PubMed and CINAHL. If any additional key terms are identified while reviewing titles and abstracts, this will be discussed with the librarian and updated. Those key terms and search strategy will be reviewed by another author and will be discussed between three persons (two authors and the librarian) if any disagreements occur. Also, we will conduct an additional manual search of the included studies. Appendix A includes a draft search strategy in PubMed and CINAHL.

\section{Eligibility criteria \\ Population}

This scoping review will consider studies that will focus on a patient with a cancer diagnosis and their unpaid caregivers. Both patients and informal caregivers who are at least 18 years of age will be included in this study. Informal caregivers who are involved in paid health service will be excluded (table 1).

\section{Outcome}

The concept of interest for the proposed scoping review is cognitive function of adults with cancer. Studies that assessed at least one of eight domains of patients' cognitive function (see 'Outcome of interest' in table 1) will be included. We will also include studies that assess patient cognitive function using their informal caregiver's report and assess caregiver characteristics using the patients' report (table 1).

\section{Concept}

Studies that were studied within the context of caregiver characteristics will be considered for inclusion in this

\begin{tabular}{|c|c|}
\hline \multicolumn{2}{|l|}{ Inclusion criteria } \\
\hline Type of publication & $\begin{array}{l}\text { Experimental, observations, } \\
\text { and qualitative studies, review } \\
\text { papers, theses and dissertation } \\
\text { conference abstracts, editorials } \\
\text { commentaries, letters and case } \\
\text { reports }\end{array}$ \\
\hline Time frame & Any \\
\hline Language & English \\
\hline Study population & $\begin{array}{l}\text { Adults with cancer and their } \\
\text { informal caregivers who } \\
\text { provided unpaid care to the } \\
\text { patients; human subjects aged } \\
\text { at least } 18 \text { years old }\end{array}$ \\
\hline Outcome of interest & $\begin{array}{l}\text { Studies that assessed at } \\
\text { least one domain of following } \\
\text { patients' cognitive function: (1) } \\
\text { attention, (2) concentration, (3) } \\
\text { information processing speed, } \\
\text { (4) memory, (5) language, (6) } \\
\text { executive function, (7) spatial } \\
\text { ability and (8) psychomotor } \\
\text { ability }\end{array}$ \\
\hline
\end{tabular}

\section{Exclusion criteria}

Studies that did not assess the relationship between informal caregiver characteristics and patients' cognitive function. Studies that were explicitly about individuals with cognitive deficits attributed to non-cancer causes such as psychiatric or neurological illness, dementia, stroke, brain injury or delirium will be excluded from the search.

scoping review. Examples of informal caregiver characteristics could include demographic information, burden of care, psychological well-being and/or the use of coping strategy (table 1).

\section{Information sources}

The following seven electronic databases will be searched: PubMed (MEDLINE), CINAHL, Embase, PsycINFO, Scopus, Sociological Abstracts and ProQuest dissertation abstracts. The search for grey literature will include the conference abstracts available through Embase, Scopus and Sociological abstracts as well as dissertations available in ProQuest dissertations.

\section{Stage 3: Study selection}

This review process will consist of two steps: (1) a title and abstract review and (2) a full-text review. Two team members will screen the title and abstract of all retrieved citations and will determine eligibility based on inclusion and exclusion criteria. On the completion of screening titles and abstracts, any disagreements will be resolved by discussion. After title and abstract review, a full-texts of included articles will be independently read by two different members. After full-text review, all three team members will meet to determine the final sample. If the relevance of an article is unclear, three team members will 
consult with a fourth team member of this research team to reach consensus. All screening will be done within an electronic screening tool.

\section{Stage 4: Charting the data}

The research team will develop data abstraction tool and determine which data should be extracted from studies to answer the research question. Three team members will independently pilot data abstraction from first 10 included studies using the data charting form and meet to confirm whether their approaches to data extraction are consistent. Questions arising when piloting the extract data form will be discussed with other team members. After piloting the form, three authors will independently record the following data from selected studies on the data charting form: (1) authors, (2) year of publication, (3) geographical setting in which the study was conducted, (4) study population (patients: age, sex and type of cancer; informal caregivers: age, sex and relationship with the patient), (5) sample size, (6) study design, (7) domains of cognitive function, (8) caregiver variables and (9) key findings (the relationship with informal caregiver characteristics and cognitive function). Might consider collecting aims of the study.

\section{Stage 5: Collating, summarizing and reporting the results}

The team will collate, summarise and report of all data obtained in stage 4 to map the knowledge on caregiver variables associated with cognitive function of adults with cancer. Using the data charting form, studies will be described by key categories, study population, domains of cognitive function, informal caregiver characteristics and its relationship. The data will be presented as tables, figures or charts. The quantitative results will be presented using the statistical findings that the studies reported such as $p$-value and correlation coefficient $(r$ value) and qualitative data from selected studies will be using qualitative methods. We will review quantitative and qualitative results separately then will compare each of result. We will use Preferred Reporting Items for Systematic Reviews and Meta-Analyses guidelines for scoping review to report results.

\section{Patient and public involvement}

Patients and public involvement were not applicable. Since this is a protocol for a scoping review, no participant recruitment will take place.

\section{ETHICS AND DISSEMINATION}

The protocol of this scoping review has been registered with Open Science Framework (see https://osf.io/fc7x9). Since the scoping review aims to synthesise information from publicly available publications, this study does not require ethics approval. The results from scoping review will be disseminated via a conference presentation and/ or the submission of a scientific journal. We anticipate the study results will provide a comprehensive knowledge of cognitive function among adults with cancer and its association with their caregiver variables. This will be necessary for cancer researchers to better understand the mechanism of cognitive function of adults with cancer and potentially develop dyadic intervention for both patient and caregiver. In turn, this understanding will allow health professionals more precisely to identify patients at risk. Policy makers can use the results to guide policy development and funding considerations.

Contributors YY developed the research question and study methods, designed the research protocol, performed the preliminary search, and contributed substantially to the drafting and editing. SR conceived of the idea of the scoping review, designed the research protocol, and contributed to the drafting and editing. AW aided in developing the systematic database search and contributed to the drafting and editing. $\mathrm{CH}$ aided in conceptualised and developing the research question and critically reviewed the manuscript. All authors have approved the final manuscript.

Funding The authors have not declared a specific grant for this research from any funding agency in the public, commercial or not-for-profit sectors.

Competing interests None declared.

Patient consent for publication Not required.

Provenance and peer review Not commissioned; externally peer reviewed.

Open access This is an open access article distributed in accordance with the Creative Commons Attribution Non Commercial (CC BY-NC 4.0) license, which permits others to distribute, remix, adapt, build upon this work non-commercially, and license their derivative works on different terms, provided the original work is properly cited, appropriate credit is given, any changes made indicated, and the use is non-commercial. See: http://creativecommons.org/licenses/by-nc/4.0/.

\section{REFERENCES}

1. Jansen CE, Miaskowski C, Dodd M, et al. A metaanalysis of studies of the effects of cancer chemotherapy on various domains of cognitive function. Cancer 2005;104:2222-33.

2. Janelsins MC, Kesler SR, Ahles TA, et al. Prevalence, mechanisms, and management of cancer-related cognitive impairment. Int Rev Psychiatry 2014;26:102-13.

3. Myers JS. Cancer- and chemotherapy-related cognitive changes: the patient experience. Semin Oncol Nurs 2013;29:300-7.

4. Von Ah D, Habermann B, Carpenter JS, et al. Impact of perceived cognitive impairment in breast cancer survivors. Eur J Oncol Nurs 2013;17:236-41.

5. Janelsins MC, Heckler CE, Peppone LJ, et al. Longitudinal trajectory and characterization of cancer-related cognitive impairment in a nationwide cohort study. J Clin Oncol 2018.

6. Merriman JD, Sereika SM, Brufsky AM, et al. Trajectories of selfreported cognitive function in postmenopausal women during adjuvant systemic therapy for breast cancer. Psychooncology 2017;26:44-52.

7. Bender CM, Merriman JD, Gentry AL, et al. Patterns of change in cognitive function with anastrozole therapy. Cancer 2015;121:2627-36.

8. Schagen SB, Boogerd W, Muller MJ, et al. Cognitive complaints and cognitive impairment following BEP chemotherapy in patients with testicular cancer. Acta Oncol 2008;47:63-70.

9. Ahles TA, Saykin AJ, Furstenberg CT, et al. Neuropsychologic impact of standard-dose systemic chemotherapy in long-term survivors of breast cancer and lymphoma. J Clin Oncol 2002;20:485-93.

10. Correa DD, Hess LM. Cognitive function and quality of life in ovarian cancer. Gynecol Oncol 2012;124:404-9.

11. Nelson CJ, Lee JS, Gamboa MC, et al. Cognitive effects of hormone therapy in men with prostate cancer: a review. Cancer 2008;113:1097-106.

12. Merriman JD, Von Ah D, Miaskowski C, et al. Proposed mechanisms for cancer- and treatment-related cognitive changes. Semin Oncol Nurs 2013;29:260-9.

13. Cimprich B, Visovatti M, Ronis DL. The attentional function Index--a self-report cognitive measure. Psychooncology 2011;20:194-202.

14. Cimprich B, So $\mathrm{H}$, Ronis $\mathrm{DL}$, et al. Pre-treatment factors related to cognitive functioning in women newly diagnosed with breast cancer. Psychooncology 2005;14:70-8. 
15. Merriman JD, Aouizerat BE, Langford DJ, et al. Preliminary evidence of an association between an interleukin 6 promoter polymorphism and self-reported attentional function in oncology patients and their family caregivers. Biol Res Nurs 2014;16:152-9.

16. Posner MI, Rothbart MK, Sheese BE. Attention genes. Dev Sci 2007;10:24-9.

17. Perrier J, Viard A, Levy C, et al. Longitudinal investigation of cognitive deficits in breast cancer patients and their gray matter correlates: impact of education level. Brain Imaging Behav 2018;46.

18. $\mathrm{Ng} \mathrm{T}$, Dorajoo SR, Cheung $\mathrm{YT}$, et al. Distinct and heterogeneous trajectories of self-perceived cognitive impairment among Asian breast cancer survivors. Psychooncology 2018;27:1185-92.

19. Cheung YT, Shwe M, Tan YP, et al. Cognitive changes in multiethnic Asian breast cancer patients: a focus group study. Ann Oncol 2012;23:2547-52.

20. Titler MG, Visovatti MA, Shuman C, et al. Effectiveness of implementing a dyadic psychoeducational intervention for cancer patients and family caregivers. Support Care Cancer 2017;25:3395-406.

21. Li Q, Loke AY. A systematic review of spousal couple-based intervention studies for couples coping with cancer: direction for the development of interventions. Psychooncology 2014;23:731-9.

22. Northouse LL, Mood DW, Schafenacker A, et al. Randomized clinical trial of a brief and extensive dyadic intervention for advanced cancer patients and their family caregivers. Psychooncology 2013;22:555-63.

23. Hayashi S, Terada S, Nagao S, et al. Burden of caregivers for patients with mild cognitive impairment in Japan. Int Psychogeriatr 2013;25:1357-63.

24. Guevara AB, Demonet J-F, Polejaeva E, et al. Association between traumatic brain injury-related brain lesions and long-term caregiver burden. J Head Trauma Rehabil 2016;31:E48-E58.

25. Barbe C, Morrone I, Novella JL, et al. Predictive factors of rapid cognitive decline in patients with Alzheimer disease. Dement Geriatr Cogn Dis Extra 2016;6:549-58.

26. Wright LK, Hickey JV, Buckwalter KC, et al. Emotional and physical health of spouse caregivers of persons with Alzheimer's disease and stroke. J Adv Nurs 1999;30:552-63.

27. lavarone A, Ziello AR, Pastore F, et al. Caregiver burden and coping strategies in caregivers of patients with Alzheimer's disease. Neuropsychiatr Dis Treat 2014;10:1407-13.
28. Tschanz JT, Piercy K, Corcoran CD, et al. Caregiver coping strategies predict cognitive and functional decline in dementia: the Cache County dementia progression study. Am J Geriatr Psychiatry 2013;21:57-66.

29. Norton MC, Clark C, Fauth EB, et al. Caregiver personality predicts rate of cognitive decline in a community sample of persons with Alzheimer's disease. The Cache County dementia progression study. Int Psychogeriatr 2013;25:1629-37.

30. Norton MC, Piercy KW, Rabins PV, et al. Caregiver-Recipient Closeness and symptom progression in Alzheimer disease. The Cache County dementia progression study. J Gerontol B Psychol Sci Soc Sci 2009;64B:560-8.

31. Peterson J, Pearce PF, Ferguson LA, et al. Understanding scoping reviews: definition, purpose, and process. J Am Assoc Nurse Pract 2017;29:12-16.

32. Northouse LL, Katapodi MC, Schafenacker AM, et al. The impact of caregiving on the psychological well-being of family caregivers and cancer patients. Semin Oncol Nurs 2012;28:236-45.

33. Hodges LJ, Humphris GM, Macfarlane G. A meta-analytic investigation of the relationship between the psychological distress of cancer patients and their carers. Soc Sci Med 2005;60:1-12.

34. Hagedoorn M, Sanderman R, Bolks HN, et al. Distress in couples coping with cancer: a meta-analysis and critical review of role and gender effects. Psychol Bull 2008;134:1-30.

35. Arksey H, O'Malley L. Scoping studies: towards a methodological framework. Int J Soc Res Methodol 2005;8:19-32.

36. Levac D, Colquhoun H, O'Brien KK. Scoping studies: advancing the methodology. Implementation Sci 2010;5.

37. Taillia H. [What's new concerning the chemobrain?]. Rev Neurol 2013;169:216-22.

38. Jim HSL, Phillips KM, Chait S, et al. Meta-Analysis of cognitive functioning in breast cancer survivors previously treated with standard-dose chemotherapy. J Clin Oncol 2012;30:3578-87.

39. Vardy J, Rourke S, Tannock IF. Evaluation of cognitive function associated with chemotherapy: a review of published studies and recommendations for future research. $J$ Clin Oncol 2007;25:2455-63.

40. East PL. Children's provision of family caregiving: benefit or burden? Child Dev Perspect 2010;4:55-61. 